\title{
NORMAL VASCULAR REACTIVITY IS RESTORED BY APIGENIN IN DIABETIC RATS
}

\author{
HANY M. EL-BASSOSSY1,2, NORA DESOKY², ABDULRAHMAN M ALAHDAL ${ }^{3}$, AHMED FAHMY2
}

1Department of Pharmacology and Toxicology, Faculty of Pharmacy, King Abdulaziz University, Kingdom of Saudi Arabia, ${ }^{2}$ Department of Pharmacology, Faculty of Pharmacy, Zagazig University, Egypt, ${ }^{3}$ Faculty of Pharmacy, Department of Clinical Pharmacy, King Abdulaziz University, Jeddah, Saudi Arabia

Email: helbassossy@kau.edu.sa

Received: 15 Dec 2016 Revised and Accepted: 22 Nov 2017

\section{ABSTRACT}

Objective: Diabetes is a disease whose complications have serious implications for the health of sufferers; one of the most serious such complications is the deterioration of vascular reactivity. Apigenin is a natural flavonoid with PKC inhibiting and antioxidant properties. In this study, the impact of apigenin on vascular reactivity deterioration was investigated.

Methods: Insulin resistance (IR) and insulin deficiency (ID) were induced by fructose and streptozotocin respectively. The isolated aortae vasoconstriction response to phenylephrine $(\mathrm{PE})$ and potassium chloride $(\mathrm{KCl})$ in addition to the vasodilation response to acetylcholine $(\mathrm{ACh})$ and sodium nitroprusside (SNP) were tested.

Results: IR and ID were associated with significantly exaggerated vasoconstriction to $\mathrm{KCl}$ and PE while significantly impaired vasodilation to ACh Response to SNP was not significantly affected by both IR and ID. In vitro incubation with apigenin $(77 \mu \mathrm{M})$ for 20 min restored normal responses to $\mathrm{PE}, \mathrm{KCl}$ and $\mathrm{ACh}$ in aortae isolated from insulin-resistant or insulin-deficient rats. Incubation for one hour with the PKC stimulant, phorbol 12myristate 13-acetate (PMA, $800 \mathrm{nM}$ ) resulted in aortic impairment similar to that seen in aortae isolated from IR and ID animals. Incubation with both apigenin prevented PMA-induced exaggerated vasoconstriction response to both PE and KCl.

Conclusion: Apigenin alleviates vascular exaggerated vasoconstriction and impaired dilation associated with diabetes or PKC activated.

Keywords: Apigenin, Diabetes, PMA, Isolated aorta, Vascular reactivity

(C) 2018 The Authors. Published by Innovare Academic Sciences Pvt Ltd. This is an open-access article under the CC BY license (http://creativecommons.org/licenses/by/4.0/) DOI: http://dx.doi.org/10.22159/ijpps.2018v10i1.16621

\section{INTRODUCTION}

Diabetes mellitus is a group of metabolic diseases resulting either from insulin resistance or insulin deficiency [1]. The former manifests as a failure of insulin to perform its intended biological function of inducing cellular absorption of glucose during its circulation [2]. In an attempt to compensate for this, hyper-insulinaemia ensures, with the eventual result that $\beta$-cells are incapable of secreting sufficient insulin, leading to type 2 diabetes [3].

Complications of diabetes have serious, deleterious effects on patients' clinical status as they generally involve major organs. However, while significant progress has been made in the treatment of the symptoms of diabetes itself, the complications remain a major cause of considerable concern. The importance of preventing the complications [4] is particularly great in the case of changes in the structure and function of the vascular system, which themselves lead to further complications [5]

Flavonoids, considered to be important in the human diet, are phenylpropanoids founds in many foods. Apigenin, a member of a non-mutagenic and less toxic [6] subclasses of flavone is found naturally in many plants. It has been reported [7] that apigenin is a powerful protein kinase C (PKC) inhibitor and antioxidant, with a range of pharmacological applications, many related to hyperlipidaemia [8] and hypertension [9].

While apigenin has reportedly induced vasodilation in the thoracic aorta of rats [10], its impact on the aorta of diabetic animals in isolated vascular preparations has yet to be confirmed. Therefore, the purpose of the current study was to investigate the effect of apigenin on the diabetes-associated exaggerated vasoconstriction and impaired vasodilation. In addition, the effect of apigenin on PKC induced vascular deterioration has been studied to investigate the apigenin effect on a major pathway of vascular complication in diabetes.

\section{MATERIALS AND METHODS}

\section{Chemicals and drugs}

Fructose was obtained from El-Nasr Chemical Company (Cairo, Egypt) and apigenin, PE, ACh and sodium nitro-prusside (SNP) from Sigma-Aldrich (Munich, Germany). ACh, SNP and PE were dissolved in distilled water. Apigenin and phorbol 12-myristate 13-acetate (PMA) were first dissolved in DMSO and then diluted to a concentration of $10 \%$ in the KHB.

\section{Animals}

In keeping with the standards of the European Convention for the Protection of Vertebrate Animals used for Experimental and Other Scientific Purposes, clear cages made from polypropylene were each used to house 3-4 male albino rats with a weight of 120-130g and obtained from Zagazig University, Zagazig, Egypt.

The rats were kept in stable environmental conditions with proportionate light-dark cycles and rodent-pellet food and purified water were supplied freely to them. Approval for the experimental protocol was obtained from the Zagazig Ethical Committee for Animal Handling. Ethical approval number is P6/4/2012.

\section{Study protocol}

24 rats were divided into experimental groups: insulin-resistant (IR) $(\mathrm{n}=8)$, insulin-deficient (ID) $(\mathrm{n}=8)$ and a control group $(\mathrm{n}=8)$. In IR rats, insulin resistance was induced by the provision of drinking water containing $10 \%$ fructose. Insulin resistance was confirmed by the presence of hyper insulinaemia (defined as $12-16 \mathrm{mg} / \mathrm{l}$ ) after six weeks. Insulin deficiency in ID rats was induced by streptozotocin (STZ, $50 \mathrm{mg} / \mathrm{kg}$, ip). Insulin deficiency was confirmed by the presence of stable hyperglycaemia $(300-350 \mathrm{mg} / \mathrm{dl})$ two weeks after STZ injection. The IR rats were left for $12 \mathrm{w}$ after fructose administration; ID rats were left for $10 \mathrm{w}$ after STZ injection. 
The descending aorta was isolated twelve hours after the final injection and placed into a cold Krebs-Henseleit buffer (KHB). The KHB contained $\mathrm{NaCl} 118.1 \mathrm{mmol}, \mathrm{NaHCO}_{3} 25.0 \mathrm{mmol}$, glucose 11.7 mmol, KCl $4.69 \mathrm{mmol}, \mathrm{CaCl}_{2} 2.5 \mathrm{mmol} \mathrm{KH}_{2} \mathrm{PO}_{4} 1.2 \mathrm{mmol}$ and $\mathrm{MgSO}_{4}$ $0.5 \mathrm{mmol}$. After clearing the aorta of connective tissue and adherent fat, it was divided into three rings, each approximately $3 \mathrm{~mm}$ in length. In order to investigate the vascular reactivity, one aortic ring was mounted in the organ bath. The impact of apigenin on vascular resistivity was investigated by first incubating the aortic rings from the rats in the three groups in apigenin $7 \mu \mathrm{M}$ for $20 \mathrm{~min}$ (conditions previous demonstrated to significantly inhibit protein kinase C [11].

\section{Vascular reactivity}

The isolated artery technique comprehensively addressed in [12-18] was applied to undertake vascular reactivity of isolated thoracic aortas. The rise in tension caused by increasing addition of phenylephrine (PE, $10^{-9}$ to $10^{-5} \mathrm{M}$ ) or $\mathrm{KCl}$ (10 to $100 \mathrm{mmol}$ ) formed the basis for the evaluation of aortic ring contraction. Meanwhile, to generate similar pre-contraction reactions in every group, the aortic rings were incubated with submaximal PE concentrations, which permitted assessment of aorta vasodilation. The organ bath was subsequently enriched with increasing concentrations of acetylcholine $\mathrm{ACh}, 10^{-9}$ to $10^{-5} \mathrm{M}$ ) or sodium nitroprusside (SNP, $10^{-9}$ to $10^{-5} \mathrm{M}$ ), with thorough documentation of responses.

For studying the effect of PMA and apigenin on vascular reactivity, PMA $(800 \mathrm{nM})$ and apigenin $(7 \mu \mathrm{M})$ were added to the organ bath during the $60 \mathrm{~min}$ equilibration period and during the addition of vasoactive agents.

\section{Statistical analysis}

Quantified values took the form of mean \pm standard error of the mean (SEM). The Prism 5 computer-based fitting program (GraphPad, CA, USA) was employed to determine the agonist maximum response (Emax) with the help of a non-linear regression. Furthermore, one-way analysis of variance (ANOVA) alongside Newman-Keuls' post-hoc test enabled estimation of statistical significance.

\section{RESULTS AND DISCUSSION}

Short-term in vitro incubation with the natural flavonoid apigenin was found to restore normal vascular reactivity in the thoracic aorta of both insulin-resistant and insulin dependent rats. This is a novel discovery. Moreover, such incubation was found to protect from the exaggerated vasoconstriction response that is induced by protein kinase C (PKC) stimulation.

This study investigated the effects of apigenin with regard to insulin resistance and deficiency-two separate processes associated with diabetes-through two well-established models $[19,20]$. Insulin deficiency was found to be associated with a reduction in vascular reactivity. The results suggest that apigenin, a flavonoid that is welltolerated by in vivo, is associated with a novel biological activity of potential use in the management of vascular complications of diabetes.

Enhanced vasoconstriction in response to both phenylephrine (PE) and $\mathrm{KCl}$ was seen was seen in the isolated aortas of diabetic rats. This was reflected in significant rises in apparent $E_{\max }(p<0.01)$ in both situations (fig. $1 \mathrm{a}$ and $1 \mathrm{~b}$ respectively and table 1 ).
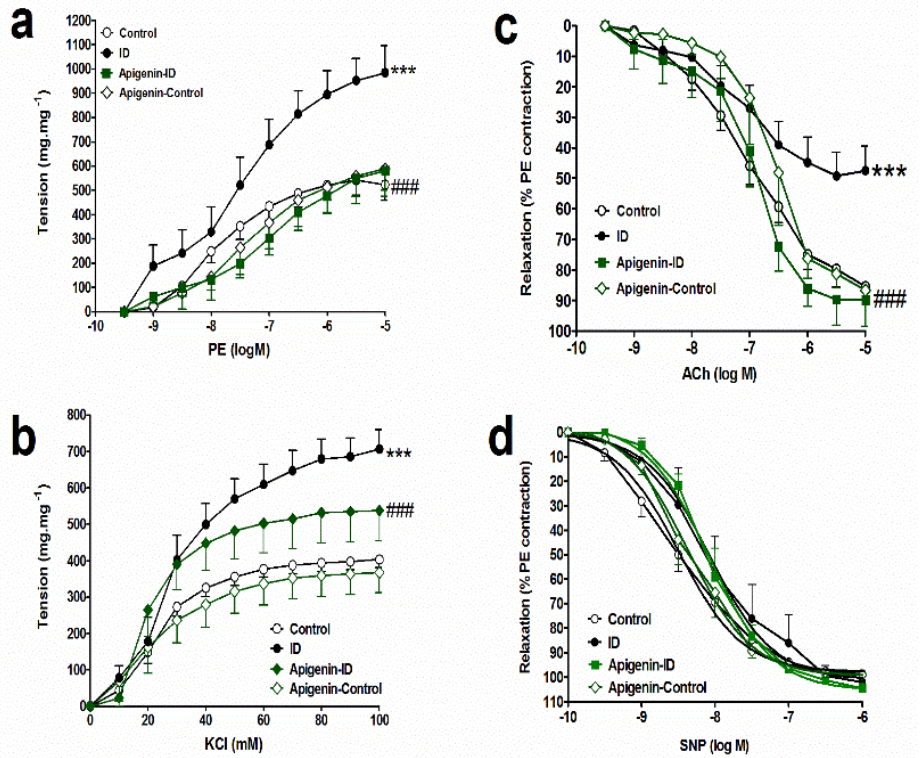

Fig. 1: Effect of apigenin incubation $(7 \mu \mathrm{M}, 20 \mathrm{~min})$ on the vasoconstriction response to (a) phenylephrine, (b) and $\mathrm{KCl}$ and the vasodilation response toACh and (d) SNP in isolated insulin deficiency (streptozotocin-induced) aortae (ID; 50 mg/dl, for 10 w). Symbols indicate mean \pm SEM for $\mathrm{N}=6-8$ animals; ${ }^{* * *} \mathrm{p}<0.001$, compared with the corresponding control group values \#\#\#p<0.001 compared with the corresponding insulin-deficient group values; by one-way analysis of variance (ANOVA) and Newman-Keuls post hoc test

Table 1: Effect of apigenin incubation $(7 \mu \mathrm{M}, 20 \mathrm{~min})$ on the maximal response $\left(\mathrm{E}_{\mathrm{max}}\right)$ and $\mathrm{pD}_{2}\left(-\mathrm{Log}_{\mathrm{EC}} \mathrm{C}_{50}\right)$ values of phenylephrine, $\mathrm{KCl}$, ACh and SNP dose-response curves of aortae isolated from streptozotocin-induced insulin deficiency (ID; $50 \mathrm{mg} / \mathrm{dl}$, for $10 \mathrm{w}$ )

\begin{tabular}{|c|c|c|c|c|c|}
\hline Treatment & & Control & Apigenin-control & ID & Apigenin-ID \\
\hline \multirow[t]{2}{*}{ PE } & $\mathbf{E}_{\max }$ & $503.5 \pm 23.5$ & $554.5 \pm 51.0$ & $936.0^{* * *} \pm 50.2$ & $555.6^{\# \# \# \pm 37.5}$ \\
\hline & $\mathrm{pD}_{2}$ & $8.0 \pm 0.2$ & $7.4 \pm 0.2$ & $7.5 \pm 0.2$ & $7.0 \pm 0.2$ \\
\hline \multirow[t]{2}{*}{ KCl } & $\mathbf{E}_{\max }$ & $363.7 \pm 8.4$ & $326.6 \pm 19.8$ & $649.7^{* * *} \pm 23.9$ & $492.7^{\# \# \# \pm 24.4}$ \\
\hline & $\mathrm{pD}_{2}$ & $-20.2 \pm 0.1$ & $-20.1 \pm 0.3$ & $-39.7^{* * *} \pm 0.3$ & 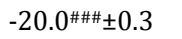 \\
\hline \multirow[t]{2}{*}{ ACh } & $\mathbf{E}_{\max }$ & $82.6 \pm 2.5$ & $90.0 \pm 5.7$ & $48.3^{* * *} \pm 4.0$ & $93.4^{\# \# \# \pm 5.2}$ \\
\hline & $\mathrm{pD}_{2}$ & $7.1 \pm 0.1$ & $6.6 \pm 0.1$ & $7.1 \pm 0.2$ & $6.9 \pm 0.1$ \\
\hline \multirow[t]{2}{*}{ SNP } & $\mathbf{E}_{\max }$ & $97.9 \pm 1.9$ & $99.5 \pm 2.3$ & $101.1 \pm 3.9$ & $105.4 \pm 1.9$ \\
\hline & $\mathrm{pD}_{2}$ & $8.4 \pm 0.1$ & $8.3 \pm 0.1$ & $8.1^{*} \pm 0.1$ & $8.1 \pm 0.1$ \\
\hline
\end{tabular}

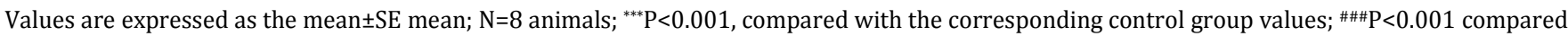
with the corresponding insulin deficient group values; by One Way ANOVA and Bonferroni post hoc test. 
In insulin-deficient rats, a significant decrease in apparent $\mathrm{E}_{\max }$ $(p<0.001)$ indicated impaired relaxation in response to $\mathrm{ACh}$ in the aortas (fig. 1c and table 1). Insulin dependency did not impact upon the in vitro response of the aortas to SNP, although its resultant hyperglycaemia did cause an exaggerated response to vasoconstriction agents [21] and impaired vasodilation [22].
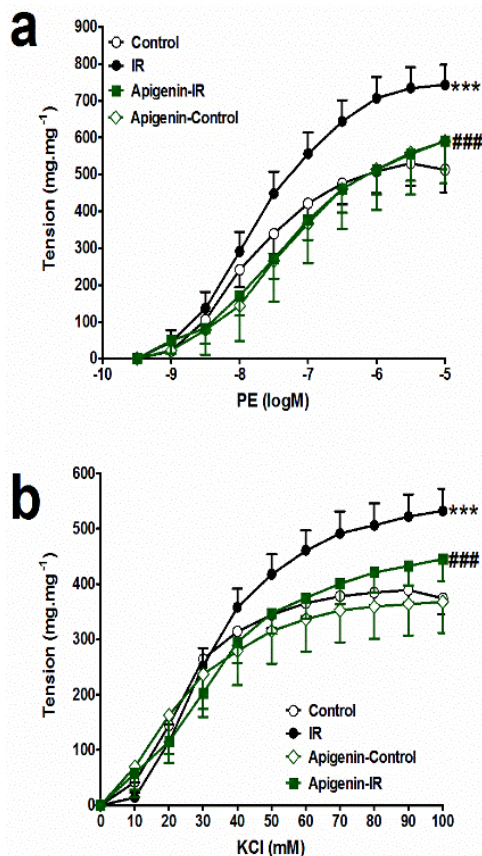

Fig. 2 and table 2 indicate the results for the insulin-resistant rats. Significant increases in the apparent $\mathrm{E}_{\max }(\mathrm{p}<0.001)$ indicated large responses both to $\mathrm{PE}$ and to $\mathrm{KCl}$ (fig. $2 \mathrm{a}$ and $2 \mathrm{~b}$ respectively). Conversely, there was a reduction in aortic response to ACh at the same significance level, as can be seen in table 2 and fig. $2 c$, in insulin-resistant rats. No connection between insulin resistance and in vitro aortic responsiveness to SNP was observed.
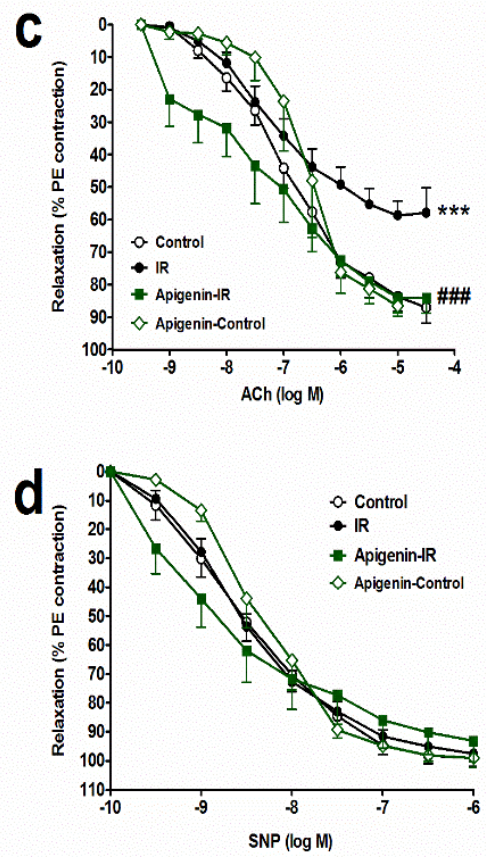

Fig. 2: Effect of apigenin incubation $(7 \mu \mathrm{M}, 20 \mathrm{~min})$ on the vasoconstriction response to (a) phenylephrine, (b) and $\mathrm{KCl}$ and the vasodilation response toACh and (d) SNP in isolated insulin resistance (fructose-induced) aortae (IR; $10 \%$ in drinking water, for $12 \mathrm{w}$ ). Symbols indicate mean \pm SEM for $N=6-8$ animals; ${ }^{* * *} \mathbf{p}<0.001$, compared with the corresponding control group values; \#\#\#p<0.001 compared with the corresponding insulin-resistant group values; by one-way ANOVA and Newman-Keulspost hoc test

Table 2: Effect of apigenin incubation $(7 \mu \mathrm{M}, 20 \mathrm{~min})$ on the maximal response $\left(\mathrm{E}_{\max }\right)$ and $\mathrm{pD}_{2}\left(-\mathrm{Log}_{\mathrm{EC}} \mathrm{C}_{50}\right)$ values of phenylephrine, $\mathrm{KCl}$, ACh and SNP dose-response curves of aortae isolated from fructose-induced insulin resistance (IR; $10 \%$ in drinking water, for $12 \mathrm{w}$ )

\begin{tabular}{|c|c|c|c|c|c|}
\hline \multicolumn{2}{|c|}{ Treatment } & Control & Apigenin-control & IR & Apigenin-IR \\
\hline \multirow{2}{*}{$\mathrm{PE}$} & $E_{\text {max }}$ & $491.8 \pm 23.1$ & $554.5 \pm 51.0$ & $731.5^{* * *} \pm 25.9$ & $551.1^{\# \# \# \pm 31.5}$ \\
\hline & $\mathrm{pD}_{2}$ & $7.9 \pm 0.2$ & $7.4 \pm 0.3$ & $7.4 \pm 0.2$ & $6.5 \pm 0.1$ \\
\hline \multirow[t]{2}{*}{$\mathrm{KCl}$} & $E_{\text {max }}$ & $352.0 \pm 8.7$ & $326.6 \pm 19.8$ & $469.8^{* * *} \pm 13.4$ & $363.6^{\# \# \# \pm 13.9}$ \\
\hline & $\mathrm{pD}_{2}$ & $-20.2 \pm 0.2$ & $-20.1 \pm 0.4$ & $-30.0^{* * *} \pm 0.15$ & 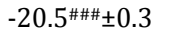 \\
\hline \multirow[t]{2}{*}{$\mathrm{ACh}$} & $E_{\text {max }}$ & $81.7 \pm 2.5$ & $89.9 \pm 5.8$ & $57.3^{* * *} \pm 2.2$ & $79.9^{\# \# \# \pm 3.9}$ \\
\hline & $\mathrm{pD}_{2}$ & $7.0 \pm 0.1$ & $7.6 \pm 0.1$ & $7.3 \pm 0.1$ & $7.2 \pm 0.2$ \\
\hline \multirow[t]{2}{*}{ SNP } & $\mathrm{E}_{\max }$ & $98.1 \pm 1.9$ & $99.6 \pm 2.4$ & $95.9 \pm 1.2$ & $88.9 \pm 3.4$ \\
\hline & $\mathrm{pD}_{2}$ & $8.5 \pm 0.1$ & $8.4 \pm 0.1$ & $8.6 \pm 0.1$ & $8.9 \pm 0.2$ \\
\hline
\end{tabular}

Values are expressed as the mean $\pm \mathrm{S}$. E mean; $\mathrm{N}=8$ animals; ${ }^{* * *} \mathrm{P}<0.001$, compared with the corresponding control group values; $\# \# \#<0.001$ compared with the corresponding insulin resistant group values; by One Way ANOVA and Bonferroni post hoc test.

As described in [3], a considerable body of evidence indicates that vascular occlusive diseases and hypertension are mediated by disease-associated deteriorated vascular reactivity in insulin resistance. Previous in vitro research has demonstrated exaggerated responses to $\mathrm{KCl}$ and $\mathrm{PE}[23]$ and impaired dilation in response to ACh [24] in the aortas of rats fed fructose. This has been reflected in reports of impaired endothelium-dependent dilation in insulindependent humans [25]. As previously mentioned, an increased influx of extracellular calcium may mediate exaggerated vasoconstriction [21]; this may explain the results seen in this study. However, vasoconstriction modulation can be the result of $\mathrm{Ca}^{2+}$ sensitization, the inhibition of myosin light chain phosphatase activity, and so independent of changes in $\mathrm{Ca}^{2+}$ within cells [26]. The inhibition of NO generation may be result in the impairment of endothelial-dependent dilation; [27] showed that NO synthase uncoupling and stimulation of reactive oxygen species (ROS) generation was associated with impaired pulmonary artery endothelial-dependent relaxation.

Fig. 1a and table 3 show a reduction in $\mathrm{E}_{\max }(\mathrm{p}<0.001)$, indicating that an exaggerated response to PE-induced by insulin deficiency was prevented by incubating the rat aorta with $7 \mu \mathrm{M}$ apigenin for 20 min. Similarly, this incubation protocol prevented an exaggerated response to $\mathrm{KCl}$-see the significant $(\mathrm{p}<0.001)$ decrease in apparent $\mathrm{E}_{\max }$ in fig. $1 \mathrm{~b}$ and table 3 . The impairment of the response to $\mathrm{ACh}$ induced by insulin resistance was alleviated $(p<0.001)$ by incubation with apigenin, shown in fig. $2 \mathrm{c}$, table 2 , although the responsiveness to SNP was affected, as indicated in fig. $2 \mathrm{~d}$. 

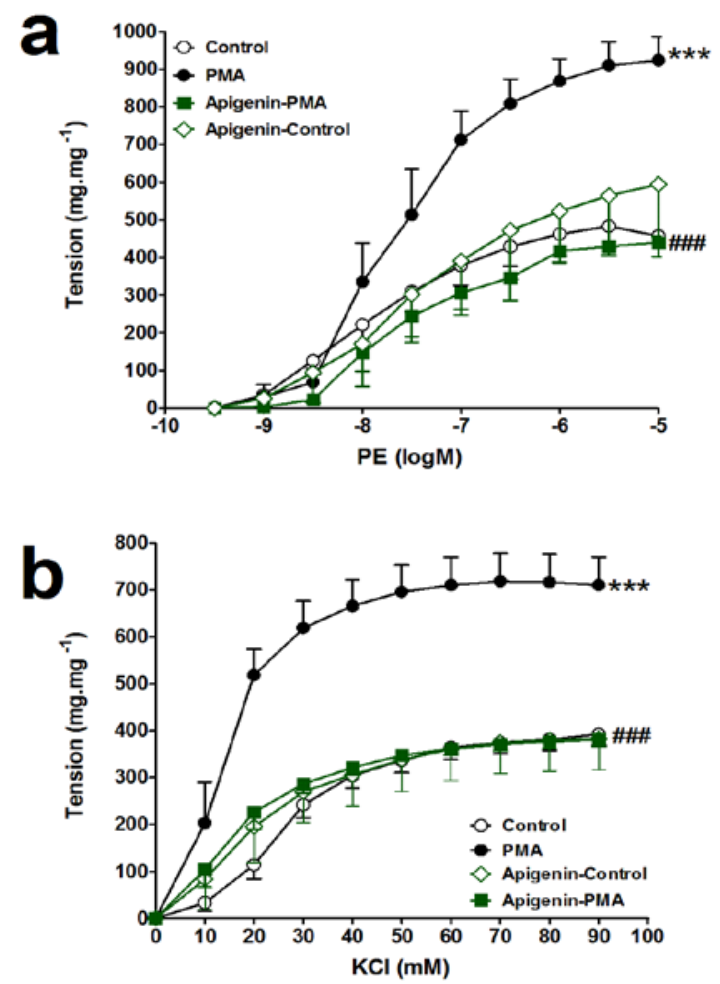

Fig. 3: Effect of apigenin incubation (7 $\mu \mathrm{M}, 20 \mathrm{~min})$ on the vasoconstriction response to (a) phenylephrine, (b) and $\mathrm{KCl}$ in injured aortae induced by PMA ( $800 \mathrm{nM}, 1$ hour) incubation. Symbols indicate mean $\pm S E M$ for $\mathbf{n}=6-8$ animals; $* * * p<0.001$, compared with the corresponding control group values; \#\#\#p<0.001 compared with the corresponding PMA group values; by one-way ANOVA and Newman-Keuls post hoc test

Table 3: Effect of apigenin incubation $(7 \mu \mathrm{M}, 20 \mathrm{~min})$ with or without PMA $(800 \mathrm{nM}, 1 \mathrm{~h})$ on the maximal response $\left(\mathrm{E}_{\max }\right)$ and $\mathrm{pD} 2(-\mathrm{Log}$ $\mathrm{EC}_{50}$ ) values of phenylephrine and $\mathrm{KCl}$ dose-response curves of isolated aortae

\begin{tabular}{|c|c|c|c|c|c|}
\hline \multicolumn{2}{|c|}{ Treatment } & Control & Apigenin-control & PMA & Apigenin-PMA \\
\hline \multirow[t]{2}{*}{ PE } & $E_{\max }$ & $442.2 \pm 21.8$ & $554.3 \pm 57.9$ & $895.8^{* * *} \pm 35$ & $416.8^{\# \# \# \pm 21}$ \\
\hline & $\mathrm{pD}_{2}$ & $8.0 \pm 0.2$ & $7.5 \pm 0.3$ & $7.7 \pm 0.1$ & $7.6 \pm 0.2$ \\
\hline \multirow[t]{2}{*}{$\mathrm{KCl}$} & $\mathrm{E}_{\max }$ & $381.2 \pm 13.8$ & $384.7 \pm 48$ & $705.7^{* * *} \pm 25$ & $378.7 \# \# \# \pm 15$ \\
\hline & $\mathrm{pD}_{2}$ & $24.7 \pm 3.1$ & $20.6 \pm 3.4$ & $12.4 \pm 4.4$ & $1.5 \pm 18.3$ \\
\hline
\end{tabular}

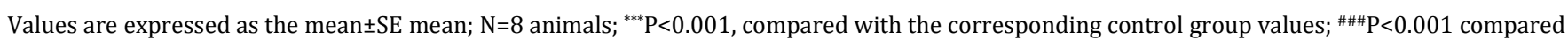
with the corresponding PMA group values; by One Way ANOVA and Bonferroni post hoc test.

In this study, the apigenin was used at that concentration at which it has previously been reported to inhibit PKC [11]. This appears to explain its direct effects on the exaggerated diabetes-related vascular contractility. The similar significant increases seen in apparent $\mathrm{E}_{\max }$ in aortic responsiveness to both $\mathrm{KCl}$ and $\mathrm{PE}$ through incubation for one hour with PMA $(800 \mathrm{nM})$ to those seen in insulin resistance and insulin deficiency supports this. However, it was also seen that protection against PMA-induced hyperresponsiveness to $\mathrm{KCl}$ or $\mathrm{PE}$ by incubation with apigenin was seen. Aortic responsiveness to ACh was unaffected by PMA and apigenin incubation (data is not shown); similarly, as illustrated by table 1 to 3 and fig. 1 to 3 , responsiveness to any of the vasoactive agents was unaffected by AP incubation. As apigenin has strong antioxidant properties [7], it will reduce the quenching of NO by suppressing ROS; apigenin is likely to mediate its effect on impaired vasodilation in diabetes, thereby restoring normal vascular dilation.

\section{CONCLUSION}

Apigenin was able to restore normal vasoconstriction and vasodilation in the thoracic aorta isolated from insulin-resistant and insulin-deficient rats. In addition, apigenin prevented PKC induced exaggerated vasoconstriction and impaired relaxation. These results point to the beneficial effect of apigenin in preventing vascular complications in diabetes most likely through protein kinase $\mathrm{C}$ inhibition.

\section{AUTHORS CONTRIBUTIONS}

HM El-Bassossy, raised the idea, designed the experiments and participated in statistical analysis and revising the manuscript; $\mathrm{N}$ Desoky, carried out laboratory experiments, animal handling procedures, data and statistical analysis and participated in drafting the manuscript; an Alahdal, participated in the study conception and manuscript revision; A Fahny, participated in study design and manuscript revision.

\section{CONFLICT OF INTERESTS}

All authors have none to declare

\section{REFERENCES}

1. Arafat M, A Salam, 0 Arafat. The association of type 2 diabetes with obesity and other factors: in the multinational community. Int J Pharm Pharm Sci 2014;6:257-60.

2. Donnelly R, X Qu. Mechanisms of insulin resistance and new pharmacological approaches to metabolism and diabetic complications. Clin Exp Pharmacol Physiol 1998;25:79-87.

3. Busija DW. Adverse effects of reactive oxygen species on vascular reactivity in insulin resistance. Antioxid Redox Signal 2006;8:1131-40.

4. Altan VM. The pharmacology of diabetic complications. Curr Med Chem 2003;10:1317-27. 
5. Akbari CM, FW LoGerfo. Diabetes and peripheral vascular disease. J Vasc Surg 1999;30:373-84.

6. Madunic J. Apigenin: a dietary flavonoid with diverse anticancer properties. Cancer Lett 2018;413:11-22.

7. Lin JK. Suppression of protein kinase $C$ and nuclear oncogene expression as possible molecular mechanisms of cancer chemoprevention by apigenin and curcumin. J Cell Biochem Suppl 1997;28-29:39-48.

8. Lestari D, EY Sukandar, I Fidrianny. In silico study of apigenin and apigetrin as an inhibitor of 3-hydroxy-3methyl-glutayl-coenzyme a reductase. Asian J Pharm Clin Res 2017;10:4.

9. Zhu ZY. Apigenin ameliorates hypertension-induced cardiac hypertrophy and down-regulates cardiac hypoxia-inducible factor-lalpha in rats. Food Funct 2016;7:1992-8.

10. Jiang HD. Endothelium-dependent and direct relaxation induced by ethyl acetate extract from flos chrysanthemi in rat thoracic aorta. J Ethnopharmacol 2005;101:221-6.

11. Zhang YH. Endothelium-dependent vasorelaxant and antiproliferative effects of apigenin. Gen Pharmacol 2000; 35: 341-7.

12. Abdallah HM. Phenolics from garcinia mangostana alleviate exaggerated vasoconstriction in metabolic syndrome through direct vasodilatation and nitric oxide generation. BMC Complementary Altern Med 2016;16:359.

13. El-Bassossy H. Ferulic acid, a natural polyphenol, alleviates insulin resistance and hypertension in fructose-fed rats: effect on endothelial-dependent relaxation. Chem Biol Interact 2016;254:191-7.

14. El-Bassossy HM, SM Abo-Warda, A Fahmy. Rosiglitazone, a peroxisome proliferator-activated receptor gamma stimulant, abrogates diabetes-evoked hypertension by rectifying abnormalities in vascular reactivity. Clin Exp Pharmacol Physiol 2012;39:643-9.

15. El-Bassossy HM, SM Abo-Warda, A Fahmy. Chrysin and luteolin attenuate diabetes-induced impairment in endothelialdependent relaxation: effect on lipid profile, AGEs and NO generation. Phytother Res 2013;27:1678-84.
16. El-Bassossy HM. Arginase inhibition alleviates hypertension in the metabolic syndrome. Br J Pharmacol 2013;169:693-703.

17. El-Bassossy HM, HA Shaltout. Allopurinol alleviates hypertension and proteinuria in high fructose, high salt and high fat-induced model of metabolic syndrome. Transl Res 2015;165:621-30.

18. Ghareib SA. 6-Gingerol alleviates exaggerated vasoconstriction in diabetic rat aorta through direct vasodilation and nitric oxide generation. Drug Des Dev Ther 2015;9:6019-26.

19. $\mathrm{Hu}$ N. Opposite effect of diabetes mellitus induced by streptozotocin on the oral and intravenous pharmacokinetics of verapamil in rats. Drug Metab Dispos 2011;39:419-25.

20. Dai S, JH McNeill. Fructose-induced hypertension in rats is concentration-and duration-dependent. J Pharmacol Toxicol Methods 1995;33:101-7.

21. Xavier FE. Time-dependent hyperreactivity to phenylephrine in aorta from untreated diabetic rats: the role of prostanoids and calcium mobilization. Vascul Pharmacol 2003;40:67-76.

22. Baluchnejadmojarad T. Beneficial effect of aqueous garlic extract on the vascular reactivity of streptozotocin-diabetic rats. J Ethnopharmacol 2003;85:139-44

23. Iyer SN, MJ Katovich. Vascular reactivity to phenylephrine and angiotensin II in hypertensive rats associated with insulin resistance. Clin Exp Hypertens 1996;18:227-42.

24. Takagawa Y. Long-term fructose feeding impairs vascular relaxation in rat mesenteric arteries. Am J Hypertens 2001;14:811-7.

25. Carmassi F. Insulin resistance causes impaired vasodilation and hypofibrinolysis in young women with polycystic ovary syndrome. Thromb Res 2005;116:207-14.

26. Morgan JP, KG Morgan. Calcium and cardiovascular function. Intracellular calcium levels during contraction and relaxation of mammalian cardiac and vascular smooth muscle as detected with aequorin. Am J Med 1984;77:33-46.

27. El-Bassossy HM. Haem oxygenase-1 induction protects against tumour necrosis factor alpha impairment of endothelialdependent relaxation in rat isolated pulmonary artery. Br J Pharmacol 2009;158:1527-35. 